\title{
FINITE DIMENSIONAL REPRESENTATIONS OF JB ALGEBRAS
}

\author{
HORST BEHNCKE
}

\begin{abstract}
Let $\Lambda$ be the set of isomorphism classes of finite dimensional JB factors, ordered by inclusion. For any $\alpha \in \Lambda$ and JB algebra $\mathcal{Q}$ denote by ${ }_{\alpha} \hat{\mathbb{Q}}$ the set of equivalence classes of factor representations of $Q$ into a JB factor of type $\alpha$. Then ${ }_{\alpha} \hat{\mathcal{A}}$ is closed.
\end{abstract}

The aim of this note is to extend the results on finite dimensional representations of $C^{*}$-algebras $[4,3.6]$ to the JB situation. The finite dimensional simple JB algebras have all been classified by Jordan, von Neumann and Wigner [6]. They are

$J_{1}$ : The real numbers $\mathbf{R}$.

$J_{2, n}$ : The spin factor over an $n$-dimensional real Hilbert space for $n \geqslant 2$.

$J_{3, k}$ : The hermitian $3 \times 3$ matrices over $\mathbf{R}(k=1), \mathbf{C}$ the complex numbers $(k=2), \mathbf{H}$ the quaternions $(k=4)$ or $\mathbf{O}$ the Cayley numbers $(k=8)$.

$J_{n, k}$ : The hermitian $n \times n$ matrices, $n \geqslant 4$, over $\mathbf{R}, \mathbf{C}$ or $\mathbf{H}$.

Let $\Lambda$ be this classifying set of finite dimensional simple JB algebras. For $\alpha, \beta \in \Lambda$ write $\alpha \leqslant \beta$ if $J_{\alpha}$ can be considered a subalgebra of $J_{\beta}$.

In this note JB algebras will not necessarily be unital and they are defined as in [3]. It is, however, possible to adjoin a unit [3]. To every pure state $\rho$ on a JB algebra $\mathbb{Q}$ one associates canonically a dense representation $\pi_{\rho}$ of $\mathbb{Q}$ into a type I JW factor $[1$, Chapter 5]. Therefore we define the primitive ideal space of $\mathscr{Q}$ by $\operatorname{Prim} \mathscr{Q}=$ $\{\operatorname{ker} \pi \mid \pi$ is a dense representation of $\mathscr{Q}$ into a type I JBW factor $\}$. With the Jacobson topology Prim $\mathscr{Q}$ is a $T_{0}$ space $[4,3.1 .3]$. It is even a $T_{1}$ space if all dense type I factor representations are finite dimensional.

As in $\left[4\right.$, Chapter 3.6] let $\hat{\mathbb{A}}_{\alpha}$, respectively $\hat{\mathbb{A}}^{\hat{\mathbb{C}}}, \alpha \in \Lambda$, denote the set of all equivalence classes of dense factor representations onto $J_{\alpha}$, respectively onto $J_{\beta}$, with $\beta \leqslant \alpha$. In this case one can identify $\hat{\mathscr{Q}}_{\alpha}$ with a part of Prim $\mathcal{Q}$. This will be done henceforth.

THEOREM. $\hat{\alpha} \hat{\mathbb{Q}}$ is closed for all $\alpha \in \Lambda$.

Proof. (a) We may assume that $\mathscr{Q}$ is unital and that $\mathscr{Q}$ has a dense set of dense representations of type $\beta$ with $\beta \leqslant \alpha$. In $C^{*}$-algebra theory the closedness of ${ }_{n} \hat{\mathbb{Q}}$ is usually achieved by the Amitsur Levitzki Theorem [4, Chapter 3.6]. It states in

Received by the editors June 22, 1982 and, in revised form, September 20, 1982.

1980 Mathematics Subject Classification. Primary 46L05.

Key words and phrases. JB algebras, finite dimensional representations. 
particular: Let $M_{n}$ be the algebra of $n$ by $n$ matrices with complex entries and for $x_{1}, \ldots, x_{r} \in M_{n}$ let

$$
P_{r}=\sum_{\sigma \in \varsigma_{r}}(\operatorname{sign} \sigma) x_{\sigma(1)} \cdots x_{\sigma(r)}
$$

Then $P_{r}=0$ for all choices of $x_{1}, \ldots, x_{r} \in M$ iff $r \geqslant 2 n$.

This result however is not available for Jordan algebras [7]. B. D. Smith has modified the concept of polynomial identities to the Jordan algebra situation and has shown in [7] a weak version of the Amitsur Levitzki result.

Let $\pi$ be a dense factor representation of $\Theta$ into $M$. Then $M$ satisfies the same standard Jordan polynomial identity as $\mathcal{Q}$, respectively $J_{\alpha}$. Because of the halving lemma [1, Theorem 6.9 and 7, Proposition 5] we see that $M$ is a finite type I JBW factor. Thus $Q$ is a type $I$ finite $\mathrm{JB}$ algebra.

(b) Let $\alpha=(n, k)$; then Lemma 6 of [2] shows that any dense representation $\pi$ of $Q$ is even of type $I_{\leqslant n} . \pi(Q)$ is finite dimensional even, because the infinite dimensional spin factor can be excluded by means of polynomial identities as in [2]. Thus $\pi(\mathbb{Q})=J_{\beta}$. Let $x_{1}, \ldots, x_{s} \in \mathbb{Q}$ such that $\pi\left(x_{1}\right), \ldots, \pi\left(x_{s}\right)$ generate $J_{\beta}$. By restricting to the subalgebra of $\mathcal{Q}$, which is generated by $x_{1}, \ldots, x_{s}$, if necessary, we may even assume $Q$ to be separable. If $\beta \leqslant \alpha$ we are finished. Thus let $\beta \$ \alpha$ and let $\pi_{i} \in{ }_{\alpha} \hat{\mathcal{Q}}$ with $\pi_{i} \rightarrow \pi$. We may assume $\left\{\pi_{i}\right\}$ to be universal with $\cap \operatorname{ker} \pi_{i}=(0)$. This net has only finitely many cluster points $\left[5\right.$, Lemma 2.4] $\rho_{1}=\pi, \rho_{2}, \ldots, \rho_{k}$. There exists an element $x \in \mathbb{Q}$ with $\pi(x) \neq 0$ and $\rho_{i}(x)=0, i=2, \ldots, k$; because $\hat{\mathscr{C}}$ is a $T_{1}$ space even. With this $x$ one constructs, by averaging of $x^{2}$, an element $e \in \mathbb{Q}$ with $\pi(e)$ the unit of $\pi(\mathscr{Q})$ and $\rho_{i}(e)=0$ for $i=2, \ldots, k$. Replacing $\Theta$ by $U_{e}(\mathscr{Q})$ if necessary, we may thus assume that $\pi$ is the only cluster point of the $\pi_{i}$. The proof can now be completed as in [5, Lemma 2.5] by using the functional calculus of continuous functions and the trace. Actually one shows that $\pi(\mathscr{Q})$ can be embedded approximately in $\pi_{i}(\mathcal{Q})$ for large $i$.

COROLlaRy 1. Let $Q$ be a JB algebra such that any dense type I factor representation is of type $\mathrm{I}_{n}$. Then Prim $\mathcal{Q}=\hat{Q}$ is locally compact.

Proof. Since for each $x \geqslant 0, \mathbb{Q} \ni \pi \rightarrow \operatorname{Tr} \pi(x)$ and $\operatorname{Tr} \pi(1-x)=n-\operatorname{Tr} \pi(x)$ are lower semicontinuous [2, Lemma 6], $\hat{\mathcal{Q}} \ni \pi \rightarrow \operatorname{Tr} \pi(x)$ is even continuous. Thus $\hat{\mathscr{Q}}$ is a $T_{2}$ space $[4,4.53]$.

Algebras of this type will be called $n$-homogeneous. The term $\alpha$-homogeneous, $\alpha \in \Lambda$, will be used in a similar fashion.

Let $\mathcal{Q}$ be an $n$-homogeneous $\mathrm{JB}$ algebra and let $P_{0} \in \hat{\mathcal{Q}}$ determine a representation of type $(n, k)$. In $J_{(n, k)}$ one can define a canonical system of matrix elements by

(i) $S_{i i}^{1}=e_{i i}, i=1, \ldots, n$,

(ii) $s_{i j}^{l}=b_{l} e_{i j}+\bar{b}_{l} e_{i j}, i \neq j, i, j=1, \ldots, n, l=1, \ldots, k, b_{1}=1$,

where the $e_{i j}$ are the usual matrix units and where $\left\{b_{l} \mid l=1, \ldots, k\right\}$ is a canonical basis of $\mathbf{R}, \mathbf{C}, \mathbf{H}$ or $\mathbf{O}$. Arguing as in [2] one can determine an open neighbourhood $U$ of $P_{0}$ and elements $s_{i j}^{l}, i, j=1, \ldots, n$ and $l=1, \ldots, k$, such that these elements satisfy the same relations at each $P \in U$ as the canonical system of matrix units. 
This shows without difficulty that Theorem 1 of [2] carries over with only minor modification to this situation. For short we write this as

COROLlARY 2. $\alpha$-homogeneous JB algebras correspond uniquely up to isomorphism to fibre bundles $\left\{\Re, p r, \hat{Q}, J_{\alpha}\right.$, Aut $\left.J_{\alpha}\right\}$.

The above results also show that locally, $n$-homogeneous algebras with $n \geqslant 4$, look like subalgebras of $(n, 4)$ homogeneous algebras. Globally this is probably not true. For $n=2,3$ a similar result holds.

\section{REFERENCES}

1. E. M. Alfsen, F. W. Schultz and E. Størmer, A Gelfand-Naimark Theorem for Jordan algebras, Adv. in Math. 28 (1978), 11-56.

2. H. Behncke and W. Bös, JB algebras with an exceptional ideal, Math. Scand. 42 (1978), 306-312.

3. H. Behncke, Hermitian Jordan Banach algebras, J. London Math. Soc. (2) 20 (1979), 327-333.

4. J. Dixmier, Les $C^{*}$-algèbres et leur représentations, Gauthier-Villars, Paris, 1964.

5. J. M. G. Fell, The dual spaces of $C^{*}$-algebras, Trans. Amer. Math. Soc. 94 (1960), 365-403.

6. P. Jordan, J. von Neumann and E. Wigner, On an algebraic generalization of the quantum mechanical formalism, Ann. of Math. (2) 35 (1934), 29-64.

7. B. D. Smith, A standard Jordan polynomial, Comm. Algebra 5 (1977), 207-218.

Department of Mathematics, University of Osnabruck, Osnabruck, Federal Republic of GERMANY 